\title{
Plasticity and Creep of Materials at Variable Stresses
}

\author{
Vladimir Andreev ${ }^{1, *}$ and Georgy Urumov $^{2}$ \\ ${ }^{1}$ Moscow State University of Civil Engineering, Yaroslavskoe shosse, 26, Moscow, 129337, Russia \\ ${ }^{2}$ North Caucasus Mining and Metallurgical Institute (State Technical University), ul. Nikolaeva 44, \\ Vladikavkaz, 362021, Russia
}

\begin{abstract}
The article deals with the modeling of elastic-plastic behavior and creep of aluminum alloys. On the basis of the treatment of equilibrium stretching diagrams and creep curves, nonlinear dependences describing the phenomena under consideration are constructed. A feature of the paper is the consideration of the creep process under varying stresses, when the growth of deformations in time is accompanied by stress relaxation.
\end{abstract}

\section{Introduction}

In this paper, we present some experimental data obtained by the authors on the plasticity and creep of an aluminum alloy. The experiments were carried out on a specially designed installation. To describe the aluminum alloy expansion diagram and curves of creep, the corresponding analytical models are constructed. The constants of the equations describing these processes are determined.

\section{Modeling plasticity and creep in the one-dimensional case}

When the rod is uniaxial stretched, its total deformation $\varepsilon$ is equal to the sum:

$$
\varepsilon=\varepsilon_{e}+\varepsilon_{0}+\varepsilon^{*},
$$

where $\varepsilon_{e}$-elastic deformation, for which Hooke's law is valid; $\varepsilon_{0}$ - "instantaneous" plastic deformation, and $\varepsilon^{*}$ - creep deformation.

Fig.1 shows equilibrium stretching diagram for AMg6 alloy.

\footnotetext{
*Corresponding author: asv@mgsu.ru
} 


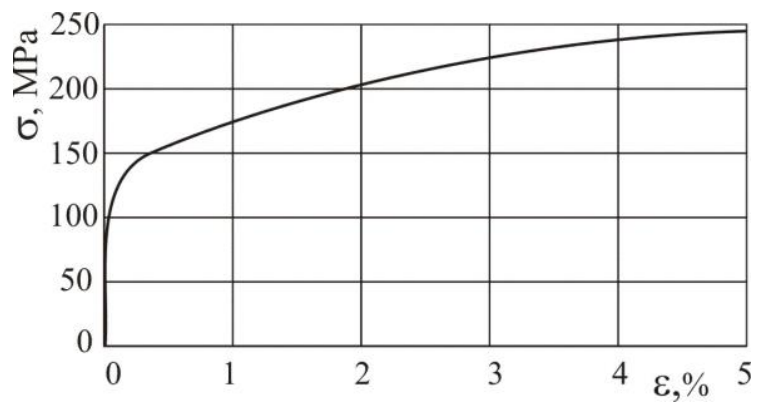

Fig.1. Equilibrium stretching diagram for $\mathrm{AMg} 6$ alloy.

In [1] various ways of approximating nonlinear diagrams are considered. In this case, a power function of the following type is sufficiently successful and at the same time simple:

$$
\sigma=A \varepsilon^{k}
$$

The parameters $A$ and $k$ can be determined from two points of the experimental diagram. For greater precision in the diagram $\sigma-\varepsilon$ several points were taken in pairs, after which averaging was carried out. For the alloy AMg6, the following data were obtained: $A=$ $477 \mathrm{MPa}, k=0.2225$.

Note that for diagrams with an explicitly expressed initial linear elastic region is better suited the function with three parameters [2 - 4]:

$$
\sigma=E-B \varepsilon^{k}
$$

As can be seen from Fig. 1 in this case there is practically no linear section and formula (2) is quite adequate.

One of the first papers devoted to the investigation of metal samples for creep and stress relaxation under uniaxial tension was work [5]. It shows that the relaxation behavior of metals at a normal temperature of uniaxial tension is sufficiently well described by the dependence

$$
\frac{d \varepsilon^{*}}{d t}=\frac{\sigma}{\eta_{0}} \exp \left|\frac{\sigma}{m}\right|,
$$

where $\eta_{0}$ - coefficient of initial relaxation viscosity, $m$ - velocity modulus. The sign of the absolute value in the exponent indicates the symmetry properties with respect to tension and compression.

Obviously, if considered "clean" creep (at constant stresses), then the equation (4) corresponds to the steady-state creep.

In Fig. 2 for the same AMg6 alloy, creep diagrams are shown for constant stresses. As can be seen, at the beginning and at the end of each diagram there are nonlinear sections. However, given that these areas are small, we can assume that equality (4) describes the diagram data quite well. 


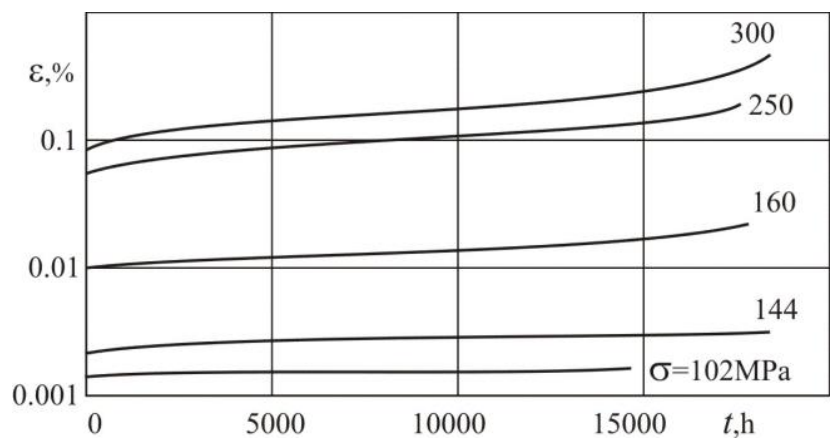

Fig. 2. Creep diagrams for AMg6 alloy.

Considering the areas of steady creep, it is possible to determine the creep deformation rates $v_{\varepsilon}^{(i)}$ for each of the diagrams, after which for any two diagrams one can obtain a system of two equations:

$$
\frac{\sigma_{1}}{\eta_{0}} e^{\frac{\sigma_{1}}{m}}=v_{\varepsilon}^{(1)} ; \quad \frac{\sigma_{2}}{\eta_{0}} e^{\frac{\sigma_{2}}{m}}=v_{\varepsilon}^{(2)}
$$

Solving this system, we find:

$$
\frac{1}{m}=\frac{1}{\sigma_{1}-\sigma_{2}} \ln \left(\frac{v_{1}}{v_{2}} \cdot \frac{\sigma_{2}}{\sigma_{1}}\right) .
$$

After this, the constant $\eta_{0}$ can be found from any of the equalities (5). After processing the experimental data, the constants in (4) were determined: $\eta_{0}=3.56 \cdot 10^{11} \mathrm{MPa} \cdot \mathrm{sec}, m=$ $167 \mathrm{MPa}$.

\section{Creep at varying stresses}

In many designs, the creep process can be accompanied by stress relaxation. The simplest example is the experience of stretching a sample on a pendulum tearing machine. The load established at the beginning of the experiment in time due to the increase in the length of the sample during creep and lowering of the pendulum, which leads to stress relaxation. Similar effect can be observed in real structures. For example, when reservoir is creeping under the influence of internal pressure, the volume of the reservoir increases, as a result of which the pressure drops.

When testing metal samples on the "Troyano" installation, the force in the sample is measured using a sufficiently rigid dynamometric ring (Fig. 3). In the process of creep, the length of the sample increases, while the diameter of the ring changes by the same amount, and the force on the sample falls. In other words, the creep process occurs with decreasing stresses. The specimen is loaded by means of vertical thrust 7 by tightening the tension nut 8 . 


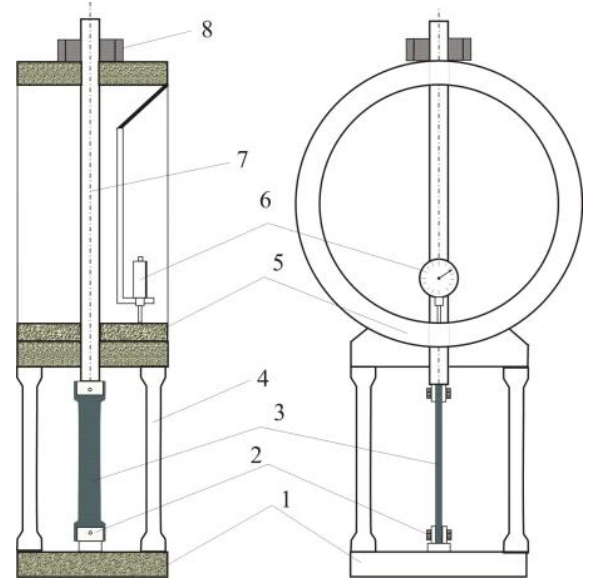

Fig. 3. Scheme of installation "Troyano",

1 - baseplates; 2 - captures; 3 - sample; 4 - support columns; 5 - dynamometric ring;

6 - indicator; 7 - vertical thrust; 8 - tensioning nut.

The magnitude of the stresses drop can be determined as follows. From the calibration curves is determined the average value of constant $b_{1}=P / \Delta l$, where $\Delta l$ - indicator reading. This constant is equal $b_{1}=3.3 \cdot 10^{6} \mathrm{MPa}$. Since the readings of the indicator $\Delta l$ are equal to the elongation of the sample, then knowing the dimensions of the sample $\left(l_{0}, F\right)$, we can calculate the magnitude of the stress drop:

$$
\Delta \sigma=P / F=b_{1} \Delta l / f=b \Delta l,
$$

where $b=b_{1} l_{0} / F$, and $\Delta \varepsilon=\Delta l / l_{0}$.

Thus, during the test, the stresses decrease in accordance with the dependence:

$$
\sigma=\sigma_{0}-b \Delta l
$$

It should be noted, that the increment of deformations of $\Delta \varepsilon$ includes both creep strains and a change in the elastic component associated with the drop in stresses.

Substituting (7) into (4) with regard to (1) and setting $\frac{d \varepsilon_{0}^{*}}{d t}=0$ we obtain a differential equation describing the creep process under consideration with decreasing stresses:

$$
\left(1+\frac{b}{E}\right) \frac{d(\Delta \varepsilon)}{d t}=\frac{\sigma_{0}-b \Delta \varepsilon}{\eta_{0}} \exp \left(\frac{\sigma_{0}-b \Delta \varepsilon}{m}\right)
$$

Using equality (7) and taking into account that $\frac{d \sigma}{d t}=-b \frac{d(\Delta \varepsilon)}{d t}$, we obtain the equation

$$
\frac{d \sigma}{d t}=-\frac{\sigma}{B \eta_{0}} \exp \left(\frac{\sigma}{m}\right)
$$

where $B=\frac{b+E}{b E}$. Separating the variables in (9) and integrating, we find 


$$
t=C-B \eta_{0} \int \frac{\exp \left(-\frac{\sigma}{m}\right)}{\sigma} d \sigma=C-B \eta_{0} F(\sigma) .
$$

In this expression the integral is denoted by $F(\sigma)$. The constant $C$ is found from the initial condition

$$
t=0, \quad \Delta \varepsilon=0 \text { or } \sigma=\sigma_{0} .
$$

Having found the constant $C$, we obtain the final solution of equation (9)

$$
t=B \eta_{0}\left[F\left(\sigma_{0}\right)-F(\sigma)\right] .
$$

The function $F(\sigma)$ appearing in (10) can be represented in the form of a series [6]:

$$
F(\sigma)=\lg \sigma+\sum_{n=1}^{\infty} \frac{\left(-\frac{\sigma}{m}\right)^{n}}{n \cdot n !} .
$$

The dependence $t(\sigma)$ according to (10) is equivalent to the dependence $\sigma(t)$, i.e., the stress relaxation curve.

In Table 1, a comparison of the experimental data reported at the "Troyano" facility is presented with the results of the theoretical calculation, based on the formula (9). In the table, for some initial stresses $\sigma_{0}$ are given values of $\sigma$, for $t=240$ hours.

Table 1. Comparison of the experimental data and theoretical calculation.

\begin{tabular}{|c|c|c|}
\hline \multirow{2}{*}{$\sigma_{0}, \mathrm{MPa}$} & \multicolumn{2}{|c|}{$\sigma, \mathrm{MPa}$} \\
\cline { 2 - 3 } & experiment & theory \\
\hline 200 & 192 & 194 \\
\hline 215 & 203 & 205 \\
\hline 230 & 219 & 218 \\
\hline
\end{tabular}

\section{Conclusions}

Taking into account the natural error in the creep tests, we can assume that the theoretical results give a satisfactory description of the experimental data.

The work is an example of a pilot study for a rather complex process of creep at variable voltages and built his theory calculation.

This work was financially supported by the Ministry of Education and Science (state task \#7.6163.2017/6.7).

\section{References}

1. P.A. Lukash, Fundamentals of nonlinear construction mechanics (Moscow, Stroyizdat, 1978)

2. V.I. Andreev, Yu.N. Malashkin, Construction mechanics and calculation of structures 6 (1983) 
3. V.I. Andreev, L.S. Polyakova, Applied Mechanics and Materials. 865 (2017)

4. V.I. Andreev, L.S. Polyakova, A.S. Avershyev, Procedia engineering 153 (2016)

5. A.L. Rabinovich, M.G. Shtarkov, E.I. Dmitrieva, On some regularities of homogeneous deformation of metals with hardening (Moscow, Oborongiz, 1959)

6. I.N. Bronstein, K.A. Semendiayev, Handbook of Mathematics for Engineers (Moscow, Nauka, 1986) 